\title{
Espacios Costeros Marinos para Pueblos Originarios: usos consuetudinarios y conservación marina
}

Marine and Coastal Spaces of Indigenous People: customary uses and marine conservation

Francisco Araos, Emilia Catalán, Ricardo Álvarez, David Núñez, Francisco Brañas y Wladimir Riquelme

\section{(2) OpenEdition Journals}

\section{Edición electrónica}

URL: https://journals.openedition.org/aa/4933

DOI: $10.4000 /$ aa. 4933

ISSN: 2357-738X

Editor

Programa de Pós-Graduação em Antropologia Social (UnB)

Edición impresa

Paginación: 47-68

ISSN: 0102-4302

\section{Referencia electrónica}

Francisco Araos, Emilia Catalán, Ricardo Álvarez, David Núñez, Francisco Brañas y Wladimir Riquelme, «Espacios Costeros Marinos para Pueblos Originarios: usos consuetudinarios y conservación marina», Anuário Antropológico [En línea], v.45 n.1 | 2020, Publicado el 16 febrero 2020, consultado el 23 julio 2022. URL: http://journals.openedition.org/aa/4933 ; DOI: https://doi.org/10.4000/aa.4933

\section{(c) (i) (9) $\Theta$}

Creative Commons - Atribución-NoComercial-SinDerivadas 4.0 Internacional - CC BY-NC-ND 4.0 https://creativecommons.org/licenses/by-nc-nd/4.0/ 


\title{
Espacios Costeros Marinos para Pueblos Originarios: usos consuetudinarios y conservación marina
}

\author{
Marine and Coastal Spaces of Indigenous People: \\ customary uses and marine conservation \\ DOI: https://doi.org/10.4000/aa.4933
}

Francisco Araos • Universidad de Los Lagos - Chile

Antropólogo Social y Doctor en Ambiente y Sociedad. Actualmente es Profesor Asociado de Universidad de Los Lagos. Intereses de investigación: dimensiones humanas de la conservación de la biodiversidad, manejo de recursos naturales, zona costera, cambio ambiental.

\section{Emilia Catalán • Investigadora Independiente - Chile}

Antropóloga Social. Forma parte del Grupo de estudios Culturas y Naturalezas de la Universidad Alberto Hurtado (Chile). Intereses de investigación: dimensiones humanas de la conservación de la biodiversidad, áreas protegidas.

Ricardo Álvarez • Universidad de Los Lagos - Chile

David Núñez • Investigador Independiente y miembro honorario Consorcio TICCA - Chile Antropólogo. Investigador Independiente. Miembro honorario Consorcio TICCA. Intereses de investigación: pueblos indígenas, prácticas y tradiciones culturales, dimensiones humanas de la conservación de la biodiversidad.

\section{Francisco Brañas • Investigadora Independiente - Chile}




\title{
Espacios Costeros Marinos para Pueblos Originarios: usos consuetudinarios y conservación marina
}

\author{
Marine and Coastal Spaces of Indigenous People: \\ customary uses and marine conservation \\ DOI: https://doi.org/10.4000/aa.4933
}

Los Espacios Costeros Marinos para Pueblos Originarios (ECMPOs) son áreas delimitadas de la zona marino-costera, cuyo objetivo es el resguardo de los usos consuetudinarios practicados por comunidades indígenas asentadas en la zona costera de Chile. Analizamos las implicancias analíticas y políticas del concepto de uso consuetudinario en el marco de los ECMPOs. Consideramos que el uso consuetudinario de un determinado espacio marino-costero se basa en una relación específica entre las comunidades indígenas con otros seres - humanos o no humanos - que se ha dado históricamente como una adecuación mutua para la reproducción de la vida, lo que representa una ética de convivencia que fundamenta formas sustentables de habitar. Tomando como ejemplo la realidad de la Región de Los Lagos, que concentra la mayor parte de las solicitudes de ECMPOs en Chile, planteamos que el establecimiento de estos espacios ha gatillado transformaciones en los diversos usos de los recursos naturales - en base a una lógica consuetudinaria -, motivando acciones que buscan la conservación de la biodiversidad en el bordemar de la zona sur austral del país.

Espacios Costeros Marinos para Pueblos Originarios. Uso consuetudinario. Comunidades indígenas. Conservación marina.
Os Espaços Costeiros Marinhos para Povos Indígenas (ECMPOs) são áreas delimitadas da zona marinho-costeira, cujo objetivo é proteger os usos costumeiros praticados pelas comunidades indígenas que habitam a zona costeira do Chile. Analisamos as implicações analíticas e políticas do conceito de uso costumeiro no âmbito de ECMPOs. Consideramos que o uso costumeiro de um espaço marinho-costeiro se baseia numa relação específica entre comunidades indígenas e outros seres - humanos e não humanos - que foi produzida historicamente como uma adaptação mútua para a reprodução da vida, o que representa uma ética da coexistência que promove modos de vida sustentáveis. Tomando como exemplo a realidade da região de Los Lagos, que concentra a maior parte das solicitações de ECMPOs no Chile, argumentamos que o estabelecimento desses espaços tem provocado transformações nos diversos usos dos recursos naturais - baseado no modelo costumeiro -, motivando ações que procuram conservar a biodiversidade no sul do país.

Espaços Costeiros Marinhos para Povos Indígenas. Uso costumeiro. Povos indígenas. Conservação marinha. 


\section{Introducción}

Chile posee una costa de más de 6000 kilómetros, que se extiende por todo el territorio continental del país, las islas y archipiélagos y la zona antártica nacional. La demanda por espacios de la zona costera para realizar múltiples actividades relacionadas con el desarrollo productivo ha sido creciente en el último tiempo, aumentando la presión sobre los ecosistemas y los modos de vida de las comunidades que dependen de los recursos del mar para su subsistencia. En este contexto, a nivel legislativo se ha promovido un ordenamiento de los usos e intereses sobre la zona costera que ha privilegiado la entrega de derechos de usos para la industria acuícola, particularmente, para la producción de salmón y mitilus en la región sur austral del país (Tecklin, 2016).

Frente a un régimen de propiedad orientado a la privatización de los recursos y espacios comunes para su usufructo en el mercado global, las comunidades locales y movimientos sociales han elaborado una serie de estrategias para enfrentar el "despojo del mar" (Bennet et al., 2015) utilizando para ello algunas de las alternativas institucionales que entregan las legislaciones ambientales e indígenas establecidas a nivel nacional e internacional (Nahuelpan, 2016; Araos, 2018)

Entre los arreglos institucionales de mayor relevancia de los últimos años en Chile, destacamos los Espacios Costeros Marinos para Pueblos Originarios (ECMPOs), precisamente, por combinar, en su constitución y posterior implementación, elementos asociados al reconocimiento de derechos de los pueblos indígenas sobre el maritorio y la conservación de la biodiversidad como consecuencia del resguardo de sus modos de vida.

Los ECMPOs se crearon el año 2008 a través de la Ley 20.249, también conocida como "Ley Lafkenche", producto de la movilización política de las comunidades indígenas mapuche lafkenche asentadas en las regiones del centro-sur y sur del país. Este movimiento etnopolítico buscó el reconocimiento legal de la relación histórica, sociocultural y ecológica de los pueblos indígenas con el mar y sus recursos naturales, a través del resguardo de sus usos consuetudinarios (De la Maza; Flores, 2012; Nahuelpan, 2016; Espinoza, 2016; Gissi et al., 2017). Para tal efecto, se establecieron los ECMPOs, áreas delimitadas de la zona marino-costera administradas por comunidades indígenas a través de un convenio de uso entregado por el Estado. Esta ley vino a cubrir una laguna en la legislación nacional que definía y autorizaba las diversas afectaciones de los espacios marinos y costeros, sin considerar la especificidad de los modos de vida de los pueblos indígenas asociados al bordemar.

El presente artículo analiza las implicancias conceptuales y políticas de la definición de los usos consuetudinarios en el marco de los ECMPOs. Particularmente, procuramos revisar cómo este arreglo institucional contrasta con las formas concretas en que se expresan los usos consuetudinarios de las comunidades indígenas, y cuál es su rol en la transición de los ECMPOs hacia la conservación de la biodiversidad. De esta forma, nos enfocaremos en los ECMPOs de la Región de Los Lagos en sus diferentes fases de tramitación, considerando que esta región de Chile concentra cerca del 70\% de las solicitudes a nivel nacional. Utilizaremos 
información disponible a la fecha en las bases de datos gubernamentales, así como entrevistas a actores claves e información documental producida por organizaciones no gubernamentales.

La argumentación se sostiene en dos hipótesis complementarias. Por una parte, consideramos que los usos consuetudinarios invocados como justificación para la creación de ECMPOs se basan en una relación específica entre las comunidades indígenas con otros - sean éstos humanos o no humanos - que se ha dado históricamente como una adecuación mutua para la reproducción de la vida. Esta lectura es lejana a la noción de los usos como prácticas ancestrales, preservadas y estáticas, como un repertorio fijo de objetos, personas y lugares, que parece ser la interpretación habitual de las autoridades gubernamentales respecto de lo consuetudinario. Y, como consecuencia de lo anterior, la segunda hipótesis plantea que el proceso de establecimiento de los ECMPOs ha gatillado transformaciones en los diversos usos de los recursos naturales, motivando acciones que buscan la conservación de la biodiversidad al interior de los espacios costeros y proyectar su desarrollo hacia la sustentabilidad.

\section{Comunidades y conservación de la biodiversidad}

A nivel mundial, se ha privilegiado un modelo de conservación de la biodiversidad - terrestre y marina - basado en la restricción de las amenazas antrópicas, mediante la instalación de áreas protegidas (Descola; Palsson, 2001, Escobar, 2008). Este modelo se ancla en la mirada occidental que postula "una dicotomía entre dos fenómenos que no son sino partes interrelacionadas de una misma totalidad: naturaleza y cultura" (Razeto; Skewes; Catalán, 2019, p. 77), siendo esta misma mirada la que ha llegado al extremo de considerar a la naturaleza como un recurso puesto a disposición del ser humano (Ingold, 2000; Latour, 2007). Este modelo ha sido impuesto a comunidades humanas, particularmente rurales e indígenas, las cuales han sido desplazadas por la instalación de áreas protegidas, y cuyas prácticas vinculadas con la biodiversidad local han sido frecuentemente prohibidas (Orlove; Brush, 1996; West, Igoe; Brockington, 2006). En estos casos, los interlocutores de las comunidades han sido el Estado, y de manera progresiva, los administradores de iniciativas privadas de conservación. Las relaciones con estos actores están caracterizadas por el conflicto, aunque en el último tiempo, se han orientado hacia la convivencia (Fraga, 2006; Ferreira et al., 2007; Simões, 2015; Rauch et al., 2019).

No obstante, la relación de las comunidades rurales e indígenas con la conservación no está dada exclusivamente por la mediación de las áreas protegidas. Extensos territorios han mantenido su biodiversidad gracias a la presencia de comunidades indígenas y rurales, desde tiempos ancestrales hasta el presente, aunque sus acciones de conservación no sean siempre explícitas (Cunha; Almeida, 2000). Este tipo de comunidades han sostenido de mejor manera aquellos conocimientos, prácticas, valores espirituales y materiales vinculados con la naturaleza, pero se encuentran lejos de ser estáticas, pues en algunos casos, hay resurgimientos o modificaciones de prácticas para enfrentar oportunidades y amenazas en 
sus territorios (Arce, Guerra; Aylwin, 2016; Araos, 2017).

Esta forma de hacer “conservación” tiene en su centro una relación particular entre comunidades humanas y otras-que-humanas. Ingold (2000), tomando como referencia principalmente a cazadores recolectores, pero también a agricultores y pastores - realidades aplicables a las comunidades costeras en cuestión en este artículo - da cuenta de la importancia de las actividades productivas de subsistencia para la comprensión de su visión su entorno, y, por tanto, de su cosmovisión. Es en la realización de estas actividades que se genera un "compromiso" ${ }^{1}$ con el entorno, donde, más que una dominación por parte de los humanos sobre la naturaleza, se trata de una relación de convivencia - no necesariamente en perfecto equilibrio en términos ecológicos -, que da cuenta de la difuminación de la mencionada dicotomía naturaleza-cultura (Descola; Palsson, 2001). En ese sentido, no corresponde una lectura donde la naturaleza es convertida en "productos" o "recursos" gracias a la intervención humana, sino que, en la convivencia con su entorno, este tipo de comunidades aportan a generar las condiciones para el florecimiento de la vida, de la que ellas también son parte. Siguiendo con la línea de interpretación de Ingold (2015), es posible observar que en la interacción entre comunidades humanas y otras-que-humanas, estas personas no realizan una "lectura de la naturaleza", como si fuera un libro del cual extraen información, sino más bien, se genera una mutua comprensión, en la cual el entorno entrega orientaciones para el comportamiento humano, que forjan una ética de la relación.

En Chile, aunque existe escasa propiedad comunitaria y pocas alternativas formales de administración común de los recursos naturales, progresivamente se organizan más "territorios o áreas conservadas por pueblos indígenas y comunidades locales", reconocidas a nivel mundial como TICCAs ${ }^{2}$, las cuales conjugan dichas prácticas y conocimientos con una gobernanza local efectiva y la conservación del territorio - ya sea de hábitats, especies, diversidad biológica, diversidad genética y servicios ecosistémicos. A diferencia de las áreas protegidas formales, los territorios conservados por comunidades cubren una amplia gama de ecosistemas y especies -nativas e introducidas -, incluyendo paisajes agrícolas, de pastoreo, caza, recolección y pesca, en espacios que conjugan valores que van desde lo productivo hasta lo sagrado (Dudley, 2008).

La realidad de comunidades costeras, comúnmente de pescadores, mariscadores y recolectores de algas, es sugerente, ya que la conservación puede emerger como parte de la gestión de los recursos marinos comunes. Existen lógicas contrapuestas respecto a la forma de manejar los recursos por parte de estas comunidades - en un polo el Estado y su legislación asociada a una lógica extractivista, en otro polo, la lógica consuetudinaria (Gelcich et al., 2006). No obstante, la administración de bienes comunes marinos, al no estar sujeta totalmente a la propiedad privada, implica una organización colectiva que debe intentar velar por la mantención y reproducción de los recursos. Las comunidades costeras chilenas regulan la explotación de los recursos del mar mediante figuras asociativas de administración de recursos marinos: algunas sujetas a la legislación pesquera como las Áreas de Manejo y Explotación de Recursos Bentónicos (AMERB)
1 El concepto original utilizado por Ingold (2000) es "engagement", el cual se ha traducido al español por los autores como "compromiso", palabra que además denota la dimensión ética involucrada en la relación en que se aplica.

2 TICCA - o ICCA en inglés - es una abreviatura para "territorios y áreas conservados por pueblos indígenas y comunidades locales". El Consorcio TICCA es una asociación internacional dedicada a promover el reconocimiento y apoyo de los TICCA. No todas las iniciativas de conservación de este tipo participan del Consorcio, no obstante, en Chile participan algunas iniciativas de conservación vinculadas con ECMPOs, como la Asociación Indígena Mapu Lahual de Butahuillimapu o la Comunidad Indígena Kawésqar residente en Puerto Edén. 
destinadas al manejo de recursos pesqueros artesanales, y otras a la legislación ambiental, como las Áreas Marinas Costeras Protegidas de Múltiple Uso (AMCP$\mathrm{MU})$, destinadas a la conservación y el uso sustentable de los recursos del mar. En este marco, los ECMPOs se han venido posicionando como una herramienta específica de manejo y conservación de los recursos y espacios marinos, destinada a los pueblos indígenas y el resguardo de sus modos de vida. Estos espacios han permitido la articulación de las comunidades con gestores públicos, científicos y organizaciones no gubernamentales, cuyo trabajo en conjunto para la protección de las prácticas consuetudinarias ha significado, en concreto, explorar el potencial conservacionista de los ECMPOs. Así, la conservación - inclusiva y orientada a las personas - no se presenta ajena, sino más bien como un elemento que viene a converger con dichas prácticas, y abrir nuevas oportunidades para la mantención de sus formas de vida (Araos; Ther, 2017).

\section{ECMPOs y usos consuetudinarios}

Las primeras reivindicaciones y demandas sociales asociadas al resguardo de los modos de vida de los pueblos indígenas de la costa emergen en el marco de la tramitación de la Ley General de Pesca y Acuicultura del año 1991, asociada a la conformación de la agrupación "Identidad Territorial Lafkenche", organización política mapuche vigente hasta la actualidad, que coordina la acción política de comunidades en la zona costera del sur de Chile, desde Arauco en la Región de Biobío hasta la Región de Los Lagos. Esta agrupación realizó la primera propuesta de Ley Lafkenche, hecho inédito en Chile, ya que se trató de un proceso político bottom-up, que significó un avance desde políticas públicas indigenistas hacia políticas públicas de reconocimiento (Gissi et al., 2017). La apuesta por una ley que diera cuenta de la relación histórica de las comunidades mapuche lafkenche con el maritorio buscaba el reconocimiento de las cosmologías de las comunidades costeras y los derechos territoriales asociados a esta relación.

En efecto, la Ley Lafkenche vela por la presencia indígena en las costas del país, por medio del resguardo del uso consuetudinario en áreas específicas de la zona marino-costera. El artículo $3^{\circ}$ de la Ley indica: "Créase el espacio costero marino de pueblos originarios, cuyo objetivo será resguardar el uso consuetudinario de dichos espacios, a fin de mantener las tradiciones y el uso de los recursos naturales por parte de las comunidades vinculadas al borde costero".

Los ECMPOs pueden recaer sobre los bienes nacionales comprendidos en el borde costero marino que se encuentran bajo la supervigilancia y administración del Ministerio de Defensa Nacional, lo que incluye playas de mar, terrenos de playa fiscales, porciones de agua y fondo, y rocas, dentro y fuera de las bahías, entre otros. La delimitación de los ECMPOs estará determinada por la superficie necesaria para asegurar el ejercicio del uso consuetudinario, reconociendo, no obstante, derechos de uso entregados con anterioridad como: Concesiones Marítimas, Áreas Aptas para la Acuicultura y Áreas de Manejo y Explotación de Recursos Bentónicos, otorgadas a titulares distintos de la comunidad o asociaciones de comunidades solicitantes. 
El concepto de uso consuetudinario toma un rol central en la Ley, pues es el objeto de protección. El resguardo lo realizan las propias comunidades y/o asociaciones de comunidades indígenas mediante un convenio de uso pactado con el Estado que les permite administrar los ECMPOs.

Según el artículo $6^{\circ}$ de la Ley los usos consuetudinarios se definen como:

Las prácticas o conductas realizadas por la generalidad de los integrantes de la asociación de comunidades o comunidad, según corresponda, de manera habitual y que sean reconocidas colectivamente como manifestaciones de su cultura. El reglamento establecerá, respecto de cada tipo de uso, la periodicidad de las prácticas o conductas. No afectará la habitualidad las interrupciones del uso. El uso consuetudinario podrá comprender, entre otros, usos pesqueros, religiosos, recreativos y medicinales.

Esta definición de uso consuetudinario fija tres criterios para considerar una práctica como consuetudinaria: (I) práctica generalizada en las comunidades, (II) práctica habitual en las comunidades y (III) que las comunidades reconozcan colectivamente dicha práctica como una manifestación de su cultura. Los dos primeros criterios son de carácter objetivo, mientras que el tercero es de carácter subjetivo, ya que depende de la definición propia de la o las comunidades (Meza-Lopehandía, 2018). El reglamento de la Ley especifica, para el primer criterio, que se considere no sólo a las personas que participen directamente de la práctica, sino también a aquellas que realizan actividades asociadas con la práctica. En el caso de la habitualidad - o periodicidad -, si bien se entiende que cada práctica tiene sus propios ciclos, se establece que debe verificarse a lo menos dos veces en un periodo de diez años, detallando que, si se trata de un uso pesquero, se exige su ejercicio uniforme en temporadas de pesca continuas al menos cada tres años.

Los criterios para dar cuenta del uso consuetudinario de un sector deben exponerse según indica el reglamento de la Ley. En primera instancia, en la solicitud de un ECMPO realizada por la comunidad o la asociación de comunidades, se deben presentar los fundamentos que justifican el uso consuetudinario del espacio solicitado, la identificación de los usos que pretendan ser incorporados en el plan de administración y un mapa sociocultural de los usos consuetudinarios invocados, el cual podrá incluir una revisión de documentación, testimonios y relatos de los ancianos y otros elementos si los hubiere. En esta etapa, aunque no se señale formalmente, es prácticamente obligatorio que las comunidades sean asesoradas por organizaciones no gubernamentales, universidades, $\mathrm{u}$ otros grupos técnicos, pues, al igual que en la etapa final cuando es necesario realizar el plan de administración del ECMPO aprobado, se deben incorporar conocimientos propios de la antropología, la geografía y la biología marina (Álvarez et al., 2018).

Una vez verificada la posible sobreposición del ECMPO con otras concesiones otorgadas a titulares distintos de las comunidades en cuestión, la Corporación Nacional de Desarrollo Indígena (CONADI) debe acreditar los usos consuetudinarios invocados. Para esto, se analizan todas las prácticas o conductas identificadas 
en la solicitud, "de forma de determinar si configuran un uso consuetudinario", tratando los siguientes puntos contemplados en el artículo $6^{\circ}$ del reglamento de la Ley: (1) Nombre de la práctica o uso consuetudinario invocado por la comunidad o asociación de comunidades indígenas solicitantes; (2) Individualización de la comunidad o comunidades indígenas que ejercen el uso consuetudinario; (3) Alcances y cobertura geográfica del uso consuetudinario; (4) Periodicidad del uso consuetudinario, habitualidad y ciclos continuos en el que se desarrolla; (5) Identificación de sitios de significación cultural; (6) Número de familias o comunidades que han ejercido el uso consuetudinario; (7) Número de familias o comunidades que actualmente ejercen el uso consuetudinario; (8) Identificación y análisis de los antecedentes que acrediten el uso consuetudinario; (9) Existencia de interrupciones que no afectan la habitualidad del uso consuetudinario y (10) Análisis y conclusiones.

De este modo se puede observar que, aunque los criterios que definen las prácticas y elementos que fundamentan el uso consuetudinario de un espacio marino-costero buscan estar en función de la realidad de cada práctica - su ciclicidad, la forma en que se realiza y el reconocimiento de la propia comunidad -, la reglamentación de la Ley limita los usos consuetudinarios a un listado de características fijas y predeterminadas, basadas en requerimientos de índole temporal y espacial. Dos cuestiones que, para las cosmologías indígenas y tradicionales, van más allá de linealidad del tiempo histórico y la bidimensionalidad del espacio geográfico.

Consideramos, por lo tanto, que es necesario revisar el concepto de uso consuetudinario, identificando las tensiones que provoca su definición institucional y, por otro lado, evaluar su potencial para el reconocimiento de derechos de acceso y uso de los territorios por parte de otras comunidades humanas.

Los usos o prácticas consuetudinarias existen y se expresan cotidianamente en muchos grupos y comunidades del país, tanto indígenas como no indígenas. No obstante, se han invisibilizado frente al poder regulatorio del Estado (Skewes, Álvarez; Navarro, 2012; Álvarez et al., 2018). La naturaleza de estos usos es más compleja que su sola puesta práctica, ya que en sí develan un intrincado tejido temporal que entrelaza múltiples tiempos - geológico, biológico, social - de las especies, dando cuenta de acuerdos, adaptaciones y aprendizajes entre las especies, que sustentan una forma particular de habitar. Una morfología "dendrita" (Skewes, Álvarez; Navarro, 2012) donde el "territorio vivido" (Ther, 2012) se co-constituye en las sinuosidades del espacio habitado. Este territorio se contrapone a lo "reticular" y rígido del espacio normado, cuyos tiempos y geografías se orientan hacia la transformación de la naturaleza en valor de cambio, como sucede con las industrias extractivas; o para su dirección y control perpetuo como lo hacen obras portuarias o viales.

Mientras que el Estado se sujeta en plataformas escritas para dar cuerpo y vida a sus regulaciones, afirmadas en una serie de estructuras político-administrativas coercitivas, que aseguran su adhesión por parte de la población, quienes aplican los usos consuetudinarios lo hacen en torno a la palabra - heredada y legitimada colectivamente- y a la confianza de la reciprocidad. Precisamente, en este último 
punto se sustenta la dimensión ética de lo consuetudinario, al situar la reciprocidad con los otros humanos y no humanos en la base de las prácticas tradicionales.

Se trata, por cierto, de una dinámica muy compleja, ya que simultáneamente existen múltiples usos en un mismo territorio: mientras algunos se dejan arrastrar íntegramente por lo normado oficialmente - que los impulsa a extraer en la medida de lo que no está prohibido por ley -, otros apelan a la costumbre y ética que heredaron de sus "mayores".

Lo cierto es que lo consuetudinario forma parte de lo subalterno, toda vez que se hace referencia a éste como una práctica “informal”, asociándose con frecuencia a modelos de vida retrógrados, anacrónicos, contrarios al desarrollo. Efectivamente los usos consuetudinarios están situados en esta compleja geometría de poder, en un extremo en el que coinciden pueblos indígenas con poblaciones rurales, de pescadores artesanales, pequeños agricultores y pastores, todas fórmulas alternativas al proyecto de desarrollo imperante, que vienen a cuestionar los altos costos socioambientales del capitalismo extractivista. Es por ello que su comprensión, estudio y fortalecimiento puede ser clave a la hora de pensar ejercicios de recomposición territorial y restauración socioambiental. Corresponde, en consecuencia, examinar los usos que se hacen de los recursos costeros a partir de los sistemas más amplios y no sólo en términos de la actividad misma. De aquí se abren posibilidades de entender, por ejemplo, la actividad pesquera como una extensión de la actividad recolectora o, incluso, agrícola, siendo necesario examinar, en términos de la realidad local, las formas en que se ha constituido el acceso al medio litoral.

\section{ECMPOs en la Región de Los Lagos y sus usos consuetudinarios}

La zona costera de la Región de Los Lagos es un territorio complejo y dinámico, sujeto a múltiples intereses económicos y políticos, tales como la industria acuícola, la pesca artesanal, el turismo, entre otros. El modelo económico neoextractivista, basado en la utilización intensiva de los recursos naturales y los servicios ecosistémicos para las actividades industriales, ha encontrado en el sur austral de Chile un espacio privilegiado para su desarrollo, impactando fuertemente en el ambiente y en las dinámicas sociales de los territorios/maritorios de esta zona del país (Claude; Oporto, 2000; Amtmann; Blanco, 2001; Buschmann; Fortt, 2005; Álvarez; Navarro 2010; Ther, 2011; Skewes, Álvarez; Navarro, 2012; Saavedra, 2013; Román et al., 2015; Álvarez, Gajardo; Ther, 2016).

En este contexto, la conservación marina, sea a través de áreas marinas protegidas o de otros instrumentos de administración marino-costera, como los ECMPOs, se ha venido posicionando como una herramienta de contestación colectiva frente a la expansión de dichas actividades económicas y sus impactos en el modo de vida de las comunidades locales. Este movimiento eco-político se ha organizado en torno de la protección de los lugares de vida de dichas comunidades, entendiéndolos como sistemas socio-ecológicos complejos que sustentan la existencia y el bienestar de un grupo humano determinado a través del tiempo (Araos, 2018).

Según datos oficiales de la Subsecretaría de Pesca, existen actualmente 89 
solicitudes de ECMPOs en el país, incluyendo un sector de la Región de Atacama y diversos sectores desde la Región del Biobío al sur, hasta la Región de Magallanes. En los últimos años se ha experimentado un importante incremento en las solicitudes de ECMPOs, pasando de 40 realizadas entre el 2009 y el 2015, a 57 realizadas entre el 2016 y 2019. Asimismo, entre el año 2016 y 2018 las solicitaciones a nivel nacional contemplaron más de 3.953 .865 hectáreas, muy por encima de las 524.000 hectáreas solicitadas en el período anterior.

\begin{tabular}{|c|c|c|c|c|c|c|c|c|c|c|c|c|c|}
\hline $\begin{array}{c}\text { Región/Año } \\
\text { solicitud }\end{array}$ & $\mathbf{2 0 0 9}$ & $\mathbf{2 0 1 0}$ & $\mathbf{2 0 1 1}$ & $\mathbf{2 0 1 2}$ & $\mathbf{2 0 1 3}$ & $\mathbf{2 0 1 4}$ & $\mathbf{2 0 1 5}$ & $\mathbf{2 0 1 6}$ & $\mathbf{2 0 1 7}$ & $\mathbf{2 0 1 8}$ & $\mathbf{2 0 1 9}$ & $\begin{array}{c}\text { total } \\
\text { solicitadas }\end{array}$ & $\begin{array}{c}\text { total } \\
\text { decretadas }\end{array}$ \\
\hline Atacama & - & - & - & - & - & - & - & - & 1 & - & - & 1 & 0 \\
\hline Araucanía & - & 1 & - & 1 & - & - & - & - & 1 & - & - & 3 & 1 \\
\hline Biobío & - & - & - & 1 & 3 & 3 & - & - & 3 & 1 & - & 11 & 0 \\
\hline Los Lagos & 1 & 7 & 5 & 1 & 4 & 1 & 8 & 16 & 11 & 11 & 5 & 70 & 9 \\
\hline Aysén & - & - & - & 1 & - & 1 & - & 1 & 2 & - & - & 5 & 0 \\
\hline Magallanes & - & - & - & - & - & - & - & - & 1 & 1 & - & 2 & 0 \\
\hline Los Ríos & - & - & - & - & - & 1 & 1 & 1 & 1 & 2 & - & 6 & 0 \\
\hline Total & 1 & 8 & 5 & 4 & 7 & 6 & 9 & 18 & 20 & 15 & 4 & 97 & 10 \\
\hline
\end{tabular}

La región de Los Lagos posee a la fecha 70 solicitudes para la creación de ECMPOs, siendo la región con más solicitudes del país. Nueve solicitudes ya cuentan con su decreto de destinación marítima y cinco de ellas han avanzado en la elaboración y aprobación de su plan de administración. Los ECMPOs en la Región de Los Lagos se distribuyen a lo largo de todo el litoral regional, desde la comuna de San Juan de la Costa por el norte, hasta la comuna de Quellón por el sur. Los nueve ECMPOs decretados son: Huentetique, Buta Lauquen Mapu y Caulin en la comuna de Ancud, Punta Capitanes en la comuna de Fresia, Bahía San Pedro, Manquemapu y Mahuidantu en la comuna de Purranque, Trincao en la comuna de Quellón y Cóndor en la Comuna de Rio Negro.
Cuadro $n^{\circ} 1$ - Solicitudes de ECMPOs por año y por región, y ECMPOs aprobadas, agosto del 2019.

Fuente: Elaboración propia en base a los datos oficiales contenidos en la tabla de atributos de la capa "Pueblos Originarios EMCPO”. Disponible en: https:// mapas.subpesca.cl/ideviewer/ 


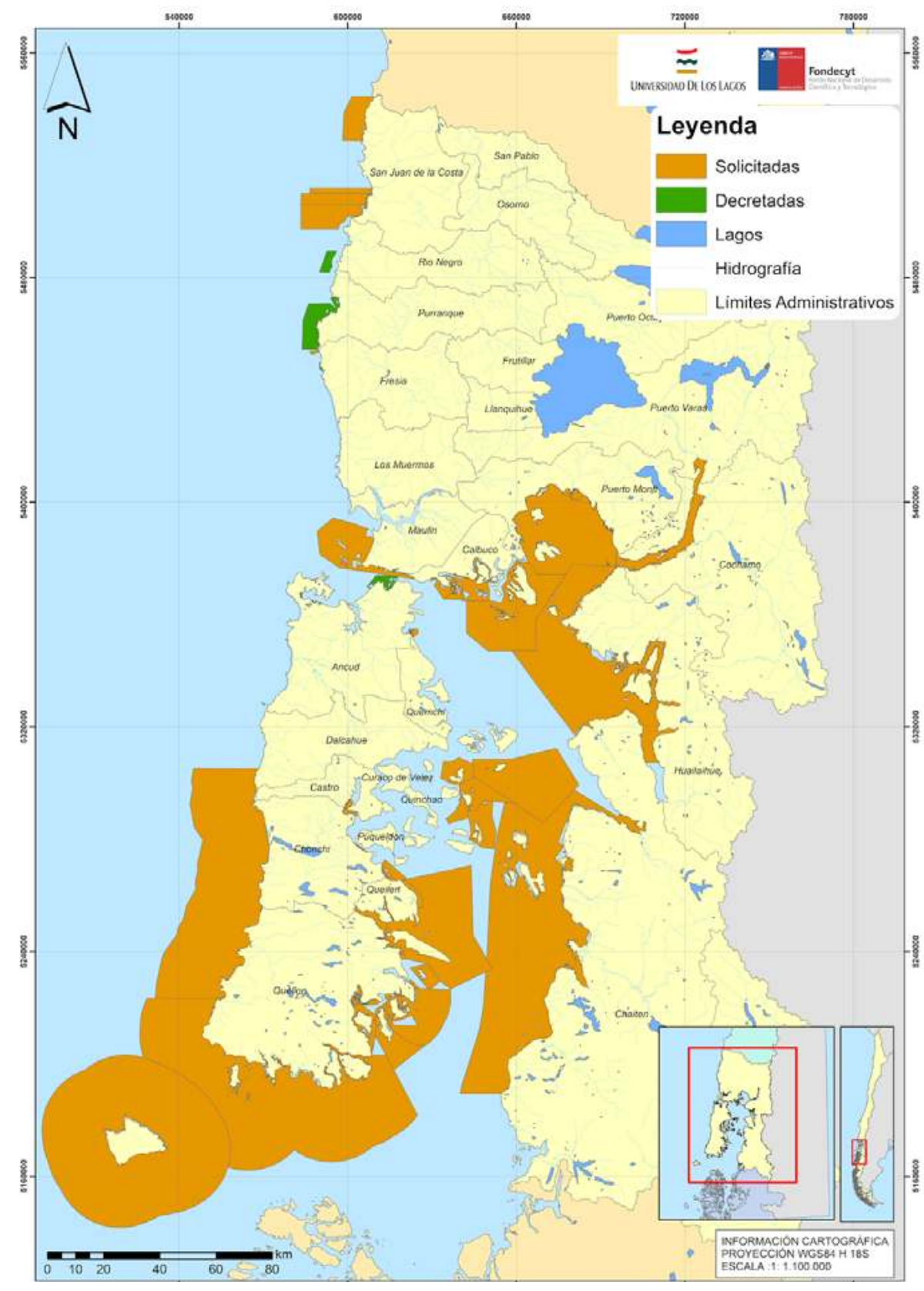

El proceso de solicitud de los ECMPOs ha permitido sistematizar las principales prácticas que se dan en los territorios marino-costeros de la zona y que se han considerado el fundamento de su uso consuetudinario. Así, por ejemplo, Sepúlveda (2017) sistematiza los componentes del uso consuetudinario del maritorio en tres comunidades indígenas del archipiélago de Chiloé, registradas durante el proceso de solicitud de ECMPOs. Entre los elementos identificados, da cuenta de lugares de especial significación, reconocidos mediante toponimia (designaciones en lengua williche, chona, castellano o combinaciones de éstas) que expresan: la presencia de elementos naturales o de los ecosistemas; lugares construidos por los antepasados que reseñan usos y prácticas ejercidos continuamente en el territorio (como corrales de pesca de piedra o varas ${ }^{3}$, cholchenes ${ }^{4}$, conchales, corrales de pirenes ${ }^{5}$ ), así como hitos reconocidos por las comunidades como puntos de
Mapa ${ }^{\circ} 1$ - ECMPOs en solicitud y decretadas en la Región de Los Lagos, Chile.

Fuente: Elaboración propia a partir de los datos de la Subsecretaría de Pesca.

3 “...técnica de pesca pasiva, donde las paredes del corral funcionaban como trampa para los peces que ingresaban en ellos en las mareas altas y se quedaban atrapados dentro del corral con las mareas bajas" (Sepúlveda 2017, p. 76)

4 Antiguos aposaderos de mariscos (Sepúlveda 2017, p. 65). 5 “...montículos de piedras apiladas de entre 20 y $60 \mathrm{~cm}$ de alto, ubicados en la línea de baja (...) En estos "corrales" Ilegan a aposarse los pilles, peces pequeños y gorditos, de gran cabeza, que desovan en estos cúmulos de piedras" (Sepúlveda 2017, p. 80). 
deslinde de su ámbito territorial.

En cuanto a las prácticas, se destacan actividades de extracción de recursos asociados a la costa y el mar, como la mariscadura, la recolección de algas, la pesca y el buceo. Estas prácticas han ido mutando en función de dos dinámicas económico-productivas que conviven conflictivamente en la zona: la tradición campesina-ribereña, caracterizada por una alta diversidad de oficios distribuidos estacionalmente y una alta movilidad espacial tierra-mar; y otra dinámica asociada a la industria pesquera-acuícola y a la pesca artesanal, asentadas en el territorio a mediados del siglo XX (Skewes, Álvarez; Navarro, 2012).

Otras prácticas asociadas al uso consuetudinario de la zona marino-costera son la navegación, la carpintería de ribera, la recolección de plantas silvestres de la costa, entre otras actividades que permiten la realización de prácticas culinarias, de salud, religiosas y recreativas (Sepúlveda, 2017). Cada una de éstas prácticas se entrelaza y ensambla territorialmente bajo lo que se ha denominado el "modelo consuetudinario" chilote, que aborda y conjuga tres esferas de la vida social en el archipiélago: la economía, la festividad y la cosmogonía (Skewes, Álvarez; Navarro, 2012; Álvarez et al., 2018).

Esto se puede observar en la mariscadura, una de las prácticas más antiguas y fundamentales para las comunidades. En términos económicos, se trata de una actividad que permite la alimentación diaria de las familias, así como la venta en espacios locales, y a su vez, se trata de una actividad comunitaria que permite que las familias locales puedan distribuirse libremente por la playa más allá de preferencias territoriales (Álvarez et al., 2018). La mariscadura es también una forma de relacionarse con los otros, ya que es una actividad cotidiana en el que vecinas y vecinos se acompañan, conversan, se ayudan, asociándose, incluso, con una dimensión festiva ya que "la convivencia en el trabajo era celebrada con la propia conversación, la comida, o el festejo propiamente tal si se daba la posibilidad de hacerlo" (Álvarez et al.,, 2018, p. 57). Destacamos también su vinculación con lo cosmogónico, entendiendo este último concepto como una comprensión del mundo que articula la relaciones entre humanos y no humanos, y que se levanta como un referencial ético para habitar el mundo. Esto implica la comprensión de fenómenos físicos y biológicos, que en el caso de la mariscadura se relacionan, por ejemplo, con el conocimiento de los ciclos de la luna asociada a las mareas y los ciclos reproductivos de las especies.

Asimismo, la recolección de mariscos se asocia a creencias y tabúes que funcionan como el encuadre de las relaciones sociales, que sanciona todo acto de egoísmo hacia los otros. En el caso de la mariscadura, esta expresión cosmogónica viene dada por la prohibición de peleas en la playa, que puedan provocan la enemistad entre las personas, el alejamiento de la vida del mar y la escasez de vida marina (Sepúlveda, 2017). También existen prohibiciones asociadas a la recolección con herramientas de metal, la utilización de caballos y carretas en la playa, lo que se puede interpretar como una forma de asegurar una extracción medida de los recursos. Otra expresión es la rogativa, por ejemplo, mediante la entrega de productos de las huertas a espíritus del mar, como la Pincoya, quien, 
en retribución, siembra la fertilidad en el mar. Una lectura desde Ingold (2015) respecto a este tipo de acciones y sanciones, consideraría que se toman orientaciones y consejos que entrega el territorio y la naturaleza, en el marco de un involucramiento comprometido con ella. Así, la ética no provendría netamente de la sociedad humana, sino más bien de la relación que se establece con el entorno. $\mathrm{Si}$ bien muchas de estas acciones ya no son realizadas masivamente por las poblaciones costeras, sus relatos evidencian las pistas de esta interacción y los valores asociados, vinculados con los usos consuetudinarios.

Como señalábamos anteriormente, consideramos que más allá de la mutabilidad de las prácticas existe una lógica subyacente a éstas, que se devela en la descripción de las múltiples esferas en que operan. Referimos a una relación específica de los humanos con otros humanos y de éstos con no humanos, orientada a la mantención de los procesos vitales que permiten la reproducción y regeneración de la vida. Postulamos que esta lógica obedece a un pacto cosmogónico entre los diversos seres que habitan el mundo - humanos y no humanos- que permite la reproducción de los bienes comunes del ambiente y la búsqueda de equidad en la distribución de sus beneficios, ya sea que esta lógica se encuentre explícita o implícita en los discursos de los actores involucrados.

Se trata de una ética relacional, que entra en tensión con la definición legal de lo consuetudinario y que se expresa bajo distintos principios, a saber: (I) integralidad del espacio y relación mar-tierra, (II) flexibilidad en el acceso y distribución de los recursos costeros y (III) asociatividad y reciprocidad (Skewes; Álvarez; Navarro, 2012).

En la Región de Los Lagos, el modelo consuetudinario se monta sobre la base de la relación especular mar-tierra, lo cual es relevante para la comprensión del paisaje y, por lo tanto, fundamental a la hora de definir los límites geográficos de los ECMPOs. Un problema generado por el encuadre temporal y espacial de la legislación y su inadecuación al territorio vivido en la práctica consuetudinaria. Situación que redunda, por ejemplo, en la imposibilidad de ejercer el resguardo de áreas esenciales en la relación mar-tierra, como los senderos de bajamar que facilitan el acceso de la comunidad a la zona costera.

El mismo argumento es válido para el resguardo de una visión integral del maritorio, versus la lógica seccionadora de las ECMPOs, que exige el mapeo del espacio al que se circunscribe el uso ancestral y la no sobreposición con concesiones ya otorgadas. Precisamente, uno de los principales conflictos en el marco de creación de los ECMPOs ha sido la desconfianza de los funcionarios y autoridades involucradas respecto a las solicitudes de espacios de grandes dimensiones, sumado a la falta de parámetros legales para determinar la adecuación de la superficie solicitada (Meza-Lopehandía, 2018).

La lógica de complementariedad entre el mar y la tierra, es relevante también para la reciprocidad que poseen las poblaciones costeras con las del interior. Los corrales de pesca, por ejemplo, si bien eran de propiedad familiar, implicaban la costumbre de que, en caso de haber abundancia de pesca, lo obtenido se repartía con la comunidad en general, incluyendo aquellas poblaciones más lejanas al mar 
(Skewes, Álvarez; Navarro, 2012). Un principio ético que puede tener una relectura al momento de establecer los acuerdos de uso entre comunidades al interior de los ECMPOs ${ }^{6}$.

Es importante relevar que el modelo consuetudinario no es un atributo exclusivo de los pueblos indígenas ya que campesinos, pescadores y recolectores, poseen cosmovisiones propias para relacionarse con los humanos y no humanos que sobreviven invisibilizadas dentro de este escenario normado. Así, por ejemplo, Sepúlveda (2017) muestra cómo las comunidades indígenas solicitantes de un ECMPO en la isla de Apiao en Chiloé reconocen que la comunidad local -humana - de ese territorio se compone también por otras comunidades no indígenas, y que el cuidado involucra a "todos", pues refiere a una formulación ética que rige las relaciones sociales y con el ambiente.

En este sentido, la relación del derecho territorial con la identidad cultural indígena se torna el punto más complejo de la visión normativa de los usos consuetudinarios, de dos maneras. Por una parte, reconocemos el riesgo de que las manifestaciones culturales de un grupo se transformen en un repertorio fijo de prácticas "legítimas" para otorgar derechos, excluyendo así otras posibilidades, una situación similar a la experimentada por las "poblaciones tradicionales" en Brasil (Cunha; Almeida, 2000; Diegues, 2000; Little, 2002; Ferreira et al., 2007). Por otra parte, observamos procesos de fortalecimiento de identidades indígenas desarrollados en el marco de conflictos socioambientales, donde la creación de ECMPOs ha aparecido como una estrategia institucional de afirmación etnopolítica (Nahuelpan, 2016; Olea; Román, 2017) que ha abierto nuevas brechas al interior de comunidades locales afectadas por los impactos de las industrias extractivas, por ejemplo, entre pescadores artesanales e indígenas de una misma localidad (Saavedra, 2013).

Han sido precisamente los recientes procesos de reconocimiento legal indígena lo que concentrado gran parte de las críticas recibidas por las comunidades solicitantes de ECMPOs - en conjunto con la extensión y su "ajuste" a los usos consuetudinarios -, particularmente por parte de la industria acuícola, con quienes mantienen disputas territoriales. Se argumenta así la instrumentalización de la identidad étnica a partir de la Ley Lafkenche, una crítica que invisibiliza las trayectorias históricas de las comunidades y exacerba la dimensión espacial de los usos consuetudinarios. Así, por ejemplo, en la Región de Los Lagos y en la Región de Aysén, la Coordinadora de Comunidades Mapuche Williche por la Defensa del Territorio "Willi Lafken Weichan" ha denunciado una serie de resquemores a solicitudes de ECMPOs por parte de la autoridad y el rubro acuícola, basados en argumentos que apelan al bajo número o la aparente inexistencia de población indígena local, situación que expone los prejuicios y operaciones políticas que tensionan el reconocimiento de derechos en el mar?

Observando dicho fenómeno desde la perspectiva planteada en este artículo, consideramos que la centralidad del concepto de uso consuetudinario en la zona marino-costera ofrece una salida convocante y extensiva a comunidades no indígenas que mantienen relaciones con otros humanos y no humanos basadas
6 Según el artículo $9^{\circ}$ del reglamento de la Ley Lafkenche, el plan de administración de un ECMPOs debe resguardar el uso del territorio por parte de comunidades indígenas y no indígenas que así lo declaren.
7 Disponible en: http://www. mapuexpress.org/wp-content/ uploads/2018/02/Declaracion-publica-oficial-Willi-Lafken -Weichan.pdf 
en una ética socioambiental consuetudinaria. Una ética que no remite sólo a las prácticas históricas, sino que también se verifica en los usos futuros que imaginan las comunidades al solicitar un ECMPO. Así, no todo lo que se quiere es mantener las tradiciones, tal como señala Sepúlveda, "lejos de eso, se está pensando el mar dentro de la posibilidad de "recuperarlo" para el futuro, y el futuro incluye perfeccionar estrategias de extracción, cultivo y comercialización de especies marinas, y la implementación de nuevas actividades como el turismo" (2017, p. 55). En esta perspectiva de futuro se integra también la conservación de la biodiversidad, como extensión de los usos consuetudinarios en el plano ético y práctico.

La convergencia de la conservación con los movimientos indígenas se ha dado de manera frecuente en el marco de procesos de solicitud de ECMPOs. El apoyo de activistas y profesionales de ONGs socioambientales ha sido clave para propiciar los informes técnicos que acompañan las solicitudes, situando a la conservación dentro de los objetivos declarados de los ECMPOs. De esta forma, más allá del apoyo técnico, vemos que el proceso de implementación de la Ley Lafkenche ha propiciado la emergencia de "ensamblajes conservacionistas", esto es,

un proceso relacional de conformación del orden social que organiza el modo de comprender y utilizar la naturaleza, y su territorialización a través de la construcción colectiva de arreglos institucionales que regulan el acceso y uso de los recursos naturales en un territorio y momento histórico determinados (Araos, 2018, p. 29).

Referimos a un proceso que se inicia con la activación de la agencia transformadora de agentes de cambio, su amplificación a través de redes y plataformas sociales, y su consolidación a través de la implementación de arreglos institucionales afines a sus propiedades emergentes. En el caso particular de los ECMPOs que han incorporado la conservación, los ensamblajes han transitado hacia modelos de conservación comunitaria, inclusiva y orientada a las personas (Vargas et al., 2016; Hiriart; Vargas, 2017; Arce; Núñez, 2017).

\section{El potencial de los usos consuetudinarios para la conservación}

La realidad de la zona marino-costera de la Región de Los Lagos, y por extensión, todo el sur austral de Chile, da cuenta de un modelo consuetudinario que no entiende el universo marítimo como separado del terrestre, por el contrario, se lo comprende como un todo integrado del cual derivan principios éticos de relación entre seres humanos y no humanos, que pueden orientar la planificación territorial, el uso sustentable del maritorio y formas inclusivas de conservación de la biodiversidad.

Planteamos que la centralidad de los usos consuetudinarios en la creación de los ECMPOs ha tenido, al menos, tres consecuencias clave para la conservación: (I) suspensión y/o paralización de concesiones marítimas para la industria acuícola en áreas prioritarias para la conservación; (II) la elaboración de una propuesta de conservación marina orientada a las personas y los modos de vida que viene 
a ampliar las políticas ambientales del país; (III) la posibilidad de extender el reconocimiento legal de los usos consuetudinarios y las medidas de protección a otros pueblos y comunidades tradicionales de Chile. Es necesario verificar cómo se manifiestan estas consecuencias con el paso del tiempo, particularmente de las últimas dos, que dependen de la aprobación e implementación de ECMPOs, situación restringida en la actualidad.

Una de las principales consecuencias concretas de la implementación de los ECMPOs es la detención en el otorgamiento de concesiones a acuicultura, infraestructura o manejo bentónico, en maritorios donde la autoridad competente ha declarado admisible la solicitud de un ECMPO, detenciones que pueden durar cerca de 5 o más años en función de la tardanza administrativa en su evaluación y declaración legal (Meza-Lopehandía, 2018). Luego de aprobada la ley, diversas comunidades indígenas fueron progresivamente solicitando ECMPOs; mapuche-lafkenche en la Región del Bío Bío y La Araucanía, mapuche williche y mapuche lafkenche en la Región de Los Lagos y de Aysén, kawéskar y yámana en la Región de Magallanes, y diaguita en el norte Chile. En efecto, la masificación de las solicitudes se relaciona, en parte, con la protección a los lugares de vida de las comunidades, que el artículo 10 de la Ley Lafkenche ${ }^{8}$ otorga frente a nuevas concesiones y proyectos industriales en el maritorio, particularmente, frente a la expansión de la salmonicultura en la región sur austral (Álvarez et al., 2018). Esta situación ha quedado plasmada también en las acciones políticas de organizaciones como "Willi Lafken Weichan", quienes han posicionado a los ECMPOs como una herramienta para ejercer el cuidado y control territorial de la zona marino-costera frente a la expansión de la salmonicultura en estas latitudes ${ }^{9}$. Tal como resume Núñez, “...las comunidades están asumiendo la defensa activa de sus territorios, una de las estrategias es la solicitud de ECMPO, pero la mirada va más allá, la idea es recuperar el mar para generar nuevas y mejores formas de trabajar y de relacionarse con el mar"10.

En cuanto al segundo punto, situamos a los ECMPOs como un arreglo institucional de la zona marino-costera que tiene el potencial para reestructurar el ordenamiento territorial, otorgándole pertinencia cultural y ambiental (Skewes, Álvarez; Navarro, 2012; Olea; Román, 2017). De la misma forma, vemos en los ECMPOs una experiencia concreta de conservación marina orientada a las personas (Vargas et al., 2016; Araos, 2017; Araos; Ther, 2017; Arce; Núñez 2017; Hiriart; Vargas, 2017). Consideramos, por lo tanto, que el aporte central de los ECMPOs a la conservación es el concepto de uso consuetudinario, pues en su ejercicio se desprende que, lejos de ser "prácticas en sí" que deban ser preservadas, son un conjunto inacabado de relaciones entre los humanos y el medio ambiente (Ingold, 2000) que, dependiendo de sus "impactos" en los sistemas socioecológicos, pueden operar como principios reguladores de la sustentabilidad en distintas realidades y coyunturas.

No necesariamente son los usos que se realizan con exactitud a la usanza antigua lo que garantizan el equilibrio con el entorno - eso implicaría además que las comunidades se congelaran en su pasado - sino que creemos que es la lógica
8 Artículo 10, Ley 20.249: "Criterios de decisión entre solicitudes incompatibles. En caso de que la misma área solicitada como espacio costero marino de pueblos originarios hubiere sido objeto de una solicitud de afectación para otros fines, se deberá suspender su tramitación hasta que se emita el informe del uso consuetudinario elaborado por la Conadi o hasta que se resuelva el recurso de reclamación que se hubiere interpuesto en su contra".

9 Disponible en: http://www. mapuexpress.org/wp-content/ uploads/2018/02/Declaracion-publica-oficial-Willi-Lafken -Weichan.pdf

10 Disponible en: https:// www.re-vuelta.cl/news/ territorialidad-indigena-y-ley-lafkenche/ 
de esas prácticas, los "pactos de convivencia" entre todos los seres que pueblan el sistema tierra los que ofrecen un camino para transitar hacia nuevas formas de habitar el planeta (Rozzi, 2012; Skewes, 2018; Araos et al., en prensa).

Este punto es de vital importancia, ya que entrega un sustento sólido para la vinculación efectiva de las comunidades locales con la protección del medioambiente, como parte del resguardo de sus modos y lugares de vida (Araos, 2017). Los usos consuetudinarios, al basarse en una visión relacional del mundo, expresan una forma de convivencia entre los seres humanos y no humanos, una ética socioambiental, cuya deriva política ofrece los lineamientos para democratizar y hacer más inclusiva la conservación de la biodiversidad.

De manera inmediata se gatilla la pregunta respecto a qué diferencia hay en el dominio de lo terrestre. Creemos que la Ley Lafkenche abre la oportunidad de extender el reconocimiento legal de los usos consuetudinarios en otros territorios. Así también está estipulado en el Convenio 169 de la OIT, que reconoce y protege el hábitat indígena, lo que incluye el derecho de esos pueblos a participar en la utilización, administración y conservación de dichos recursos naturales presentes en su hábitat. La Ley Lafkenche es lo más cercano a incorporar al derecho nacional el concepto de "territorio indígena" estableciendo expresamente facultades de los pueblos originarios en la utilización, administración y conservación de los recursos naturales existentes en dicho espacio (Hervé; Pérez, 2012, p. 72, en Meza-Lopehandía, 2018, p. 11). Empero, es necesario comprender que la lógica de propiedad privada en el ámbito terrestre se encuentra más arraigada que en el ámbito marino-costero, que corresponden a bienes públicos que se concesionan, por lo que una legislación similar a la Ley Lafkenche se abre, como primera posibilidad, en espacios de bienes públicos, como las áreas silvestres protegidas.

Como ya se señaló, la idea de uso consuetudinario de un territorio interpela a otros pueblos y comunidades tradicionales de Chile, ya que su conceptualización, incluso la expuesta en la Ley Lafkenche, aplica para muchas realidades no indígenas. Por ejemplo, los arrieros de la zona central, cuya relación con el territorio se funda en la crianza de animales foráneos - vacunos, caballares, caprinos, entre otros -, se vinculan entre ellos, con los animales, con el ambiente montañoso, el matorral y bosque nativo bajo una lógica consuetudinaria, heredada por sus ancestros, poseedor de una visión integral del territorio como un espacio común. Dichas relaciones establecen a la práctica arriera como tendiente a la conservación (Catalán, 2015; Suckel; Razeto, 2016; Razeto, Skewes; Catalán, 2019). O los recolectores de algas de la Región de O’Higgins, quienes en sucesivas generaciones han ido habitando la zona costera - conocida como la orilla -, estableciendo un territorio orientado a la explotación de recursos del intermareal, por medio de prácticas de manejo tradicional de los recursos de mar (Araos, 2015). Grupos que han manifestado, además, su ética de cuidado ambiental por medio de la creación de un Santuario de la Naturaleza para la protección de un bosque de macroalgas (Araos; Ferreira, 2013; Gelcich et al., 2015). Esta lógica nos acerca a la posición de Ingold (2015), quien critica la visión esencialista detrás del concepto de "lo indígena", que utiliza como criterio fundante la idea de las geologías - son 
indígenas aquellos descendientes de las personas que estaban en un territorio previo a la llegada de colonizadores -, mientras apuesta por un enfoque relacional para identificar a este tipo de comunidades, en función de su experiencia de vinculación con la tierra.

\section{Conclusión}

La creación e implementación de Espacios Costeros Marinos para Pueblos Originarios ha puesto en evidencia las tensiones territoriales que se viven a diario en la zona costera de Chile. El aumento significativo de solicitudes en el sur austral revela el conflicto latente entre las comunidades locales y la industria acuícola, la descomposición del ideal de progreso levantado por el Estado y las empresas, y las estrategias políticas para enfrentar su expansión e impactos socioambientales.

No obstante, más allá de la controversia, vemos que los ECMPOs representan una oportunidad para repensar las formas de habitar los territorios/maritorios, basados en el uso consuetudinario ejercido por comunidades indígenas y tradicionales sobre la base de una ética de convivencia entre humanos y no humanos. Un nuevo punto de partida que invita a reorientar la conservación y la sustentabilidad de los maritorios hacia el resguardo de los procesos vitales de los sistemas socioecológicos y su regeneración cuando se encuentren dañados.

De esta forma, acreditamos que tanto la expansión de los ECMPOs en la Región de Los Lagos y en el sur austral de Chile, como su orientación hacia la conservación marina, dan cuenta de la incubación de un proceso de transición socio-ecológica en marcha, que tiene el potencial de incentivar una transformación del modo de comprender, habitar y usar la zona marino-costera y sus recursos naturales. 


\section{Referencias}

ÁLVAREZ, Catalina; GAJARDO, Claudio; THER, Francisco. Actores y conflictos territoriales en una figura de administración pública de la pesca artesanal: el caso de la zona contigua en las regiones de Los Lagos y de Aysén, sur de Chile. Magallania, v. 44, n. 1, p. 131-147, 2016.

ÁLVAREZ, Ricardo; NAVARRO, Magdalena. Conflictos asociados a los múltiples usos. En: HUCKE-GAETE, Rodrigo; LO MORO, Pablo; RUIZ, Jorge (Eds.). Conservando el mar de Chiloé, Palena y las Guaitecas: síntesis del estudio Investigación para el desarrollo de Área Marina Costera Protegida Chiloé, Palena y Guaitecas. Puerto Montt: Gobierno Regional de Los Lagos, 2010. p. 125-143.

ÁLVAREZ, Ricardo; THER, Francisco; ARAOS, Francisco; HIDALGO, Carlos. Aproximaciones hacia el Uso Consuetudinario para el contexto insular austral. Fogón - Revista Internacional de Estudio de las Tradiciones, v. 1, n. 2. p. 53-64, jul.-dic. 2018.

AMTMANN, Carlos; BLANCO, Gustavo. Efectos de la Salmonicultura en las Economías Campesinas de la Región de Los Lagos, Chile. Revista Austral de Ciencias Sociales, n. 5, p. 93-106, 2001.

ARAOS, Francisco. Navegando en aguas abiertas: tensiones y agentes en la conservación marina en la Patagonia chilena. Revista de Estudios Sociales, n. 5, p. 27-41, 2018.

ARAOS, Francisco. Más allá de la biodiversidad: aportes de la antropología a la conservación marina en Chile". Revista Austral de Ciencias Sociales, n. 33, p. 21-35, 2017.

ARAOS, Francisco. Habitando la orilla: La recolección de algas en el litoral central de Chile. Espacio Regional Revista de Ciencias Sociales, v. 2, n. 12, p. 137-151, 2015.

ARAOS, Francisco; FERREIRA, Lucía da Costa. The construction of an environmental arena for marine conservation in Chile. Ambiente e Sociedade, v. 16, n. 13, p. 119-136, 2013.

ARAOS, Francisco; THER, Francisco. How to adopt an inclusive development perspective for marine conservation: preliminary insights from Chile. Current Opinion in Environmental Sustainability, n. 2, p. 68-72, 2017.

ARAOS, Francisco; RIQUELME, Wladimir; SKEWES, Juan Carlos; VIANNA, Ana Beatriz; ÁLVAREZ, Ricardo; THER, Francisco; IWAMA, AllanYu; ALBAGLI, Sarita; COSTA, Aderval; DUARTE, Emmanuel. La vida después de la devastación: lo común de la tragedia en territorios socio y biodiversos de Chile y Brasil. Revista de Antropologías del Sur. (En prensa).

ARCE, Lorena; GUERRA, Felipe; AYLWIN, José (Eds.). Cuestionando los enfoques clásicos de conservación en Chile: el aporte de los pueblos indígenas y las comunidades locales a la protección de la biodiversidad. Temuco: Observatorio Ciudadano/Consorcio TICCA, 2016.

ARCE, Lorena; NÚÑEZ, David. Informe de Participación Congreso Internacional de Áreas Marinas Protegidas IMPAC4. 2017. Disponible en: https://www.iccaconsortium.org/ wp-content/uploads/2017/10/Participacio\%CC\%81n-C-TICCA-en-IMPAC4.pdf. Acceso el: 09 mar. 2019.

BENNETT, Nathan James; GOVAN, Hugh; SATTERFIELD, Terre. Ocean Grabbing. Marine Policy, n. 57, p. 61-68, 2015.

BUSCHMANN, Alejandro; FORTT, Antonia. Efectos ambientales de la acuicultura intensiva y alternativas para un desarrollo sustentable. Revista Ambiente y Desarrollo, v. 21, n. 3, p. 58-64, 2005. 
Francisco Araos, Emilia Catalán, Ricardo Álvarez, David Núñez, Francisco Brañas, Wladimir Riquelme

CATALÁN MARTINA, Emilia. Relaciones humano-ambiente en el Parque Nacional La Campana: una trayectoria de encuentros y desencuentros entre comunidades locales y el área protegida. Memoria (para optar al título profesional de Antropóloga Social) Facultad de Ciencias Sociales, Universidad de Chile, Santiago, 2015.

CUNHA, Manuela Carneiro da; ALMEIDA, Mauro W. B. Indigenous people, traditional people, and conservation in the Amazon. Daedalus Journal of the American Academy of Arts and Sciences, v. 129, n. 2, p. 315-338, 2000.

CLAUDE, Marcel; OPORTO, Jorge. La ineficiencia de la salmonicultura en Chile: aspectos sociales económicos y ambientales. Santiago: Fundación Terram, 2000. Disponible en: http://www.terram.cl/wp-content/uploads/2014/08/rpp1_julio2000.pdf. Acceso el: 09 abr. 2019.

DELAMAZA, Gonzalo; FLORES, Fabián. Incidencia del movimiento indígena en la ley que crea el espacio costero marino de los pueblos originarios en Chile. En: FERNÁNDEZ, Margarita; SALINAS, Javier (Comps.). Defensa de los derechos territoriales en Latinoamérica. Santiago: RIL Editores, 2012. p. 93-128.

DESCOLA, Phillipe; PALSSON, Gisli (Eds.). Naturaleza y sociedad: perspectivas antropológicas. Trad. de Stella Mastrangelo. México: Siglo XXI editores, 2001.

DIEGUES, Antonio Carlos (Org.). Os saberes tradicionais e a biodiversidade no Brasil. São Paulo: MMA/COBIO/NUPAUB/USP, 2000.

DUDLEY, Nigel. Directrices para la aplicación de las categorías de gestión de áreas protegidas. Gland, Suiza: UICN, 2008.

ESCOBAR, Arturo. Territories of difference: place, movements, life, redes. Durham: Duke University Press, 2008.

ESPINOZA, Claudio. Ley del borde costero y cuestión étnica en Chile: del discurso a la práctica política. Universum, Talca, v. 3, n. 1, p. 123-139, 2016.

FERREIRA, Lucía da Costa; VIERA DE CAMPOS, Simone; JUNQUEIRA, Eliana; VIANNA, Ana Beatriz; CAROSPRESO, Camilo. Encontro das águas: dinâmicas sociais e biodiversidade na Amazônia brasileira. Teoría \& Pesquisa, v. 16, n. 1, p. 15-37, 2007.

FRAGA, Julia. Local perspectives in conservation politics: the case of the Ría Lagartos Biosphere Reserve, Yucatán, México. Landscape and Urban Planning, v. 74, n. 3-4, p. 285-295, 2006.

GELCICH, Stephan; EDWARDS-JONES, Gareth; KAISER, Michel J.; CASTILLA, Juan C. Co-management policy can reduce resilience in traditionally managed marine ecosystems. Ecosystems, v. 9, n. 6, p. 951-966, 2006.

GELCICH, Stephan; PERALTA, Leonardo; DONLAN, C. Josh; GODOY, Natalio; ORTIZ, Verónica; TAPIA-LEWIN, Sebastián; GODOY, Francisco. 2015. Alternative strategies for scaling up marine coastal biodiversity conservation in Chile. Maritime Studies, v. 14, n. 5, p. 1-13, 2015.

GISSI, Nicolás; IBACACHE, Daniela; PARDO, Bernardo; ÑANCUCHEO, Maria Cristina. El Estado chileno, los lafkenche y la Ley 20.249: ¿Indigenismo o política del reconocimiento?. Revista Austral de Ciencias Sociales, n. 32, p. 5-21, 2017.

HIRIART-BERTRAND, Luciano; VARGAS, Carlos I. Towards the implementation of new right-based management for coastal indigenous communities in Chile. Abstract book 28th International Congress for Conservation Biology (ICCB). 2017. Disponible en: https://conbio.org/mini-sites/iccb-2017. Acceso el: 09 abr. 2019.

INGOLD, Tim. Soñando con dragones: sobre la imaginación de la vida real. Trad. de 
Francisco Araos, Emilia Catalán, Ricardo Álvarez, David Núñez, Francisco Brañas, Wladimir Riquelme

Santiago Restrepo. Nómadas, n. 42, p. 13-31, 2015.

INGOLD, Tim. The perception of the environment: essays on livelihood, dwelling and skill. London: Routledge, 2000.

LATOUR, Bruno. Nunca fuimos modernos: ensayo de Antropología Simétrica. México: Siglo XXI, 2007.

LITTLE, Paul E. Territórios sociais e povos tradicionais no Brasil. Anuário Antropológico, v. 28, n. 1, p. 251-290, 2002.

MEZA-LOPEHANDÍA, Matías. La Ley Lafkenche: análisis y perspectivas a 10 años de su entrada en vigor. Asesoría técnica parlamentaria, documento elaborado para la Comisión de Vivienda, Desarrollo Urbano y Bienes Nacionales. Biblioteca del Congreso Nacional de Chile BCN. 2018. Disponible en: https://www.bcn.cl/obtienearchivo?id=repositorio/10221/25431/1/BCN___FINAL__La_Ley_Lafkenche_10_anos_despues_2018.pdf. Acceso el: 09 abr. 2018.

NAHUELPAN, Héctor. Micropolíticas mapuche contra el despojo en el Chile neoliberal. La disputa por el lafkenmapu (territorio costero) en Mehuín. Izquierdas, v. 30, p. 89123, 2016.

OLEA, Jorge; ROMÁN, José. Ordenamiento territorial y modernización en la Patagonia norte chilena - el caso de la comuna de Hualaihué: borde costero, salmoneras y comunidades indígenas. Planeo, 70, p. 1-11, 2017.

ORLOVE, Benjamin; BRUSH, Stephen. Anthropology and the conservation of biodiversity. Annual Review of Anthropology, v. 25, p. 329-352, 1996.

RAUCH, Marcos; CATALÁN, Emilia; MARTínEZ, Paula; AGUILERA, Guido; VALENZUELA, Ivonne; MALDONADO, Sandro. Gestión intercultural para la conservación en Áreas Silvestres Protegidas del Estado: aprendizajes y desafíos. Revista Austral de Ciencias Sociales, n. 3, p. 183-204, 2019.

RAZETO, Jorge; SKEWES, Juan Carlos; CATALÁN, Emilia. 2019. Prácticas de conservación, sistemas naturales y procesos culturales: apuntes para una reflexión crítica desde la etnografía. En: CERDA, Claudia; SILVA-RODRÍGUEZ, Eduardo; BRICEÑO, Cristóbal (Eds.). Naturaleza en sociedad: una mirada a la dimensión humana de la conservación de la biodiversidad. Santiago: Ocho Libros Editores, 2019. p. 75-106.

ROMÁN, Álvaro; BARTON, Jonathan; BUSTOS, Beatriz; SALAZAR, Alejandro (Eds.). Revolución salmonera: paradojas y transformaciones territoriales en Chiloé. Santiago: RIL Editores; Instituto de Estudios Urbanos y Territoriales UC, 2015.

ROZZI, Ricardo. Biocultural ethics: the vital links between the inhabitants, their habits and regional habitats. Environmental Ethics, n. 34, p. 27-50, 2012.

SAAVEDRA, Gonzalo. La pesca artesanal en las encrucijadas de la modernización: usos, apropiaciones y conflictos en el borde costero del sur de Chile. Revista Andaluza de Antropología, n. 4, p. 79-102, 2013.

SEPÚLVEDA, Carolina. 2017. Dimensiones de valor del espacio marino en Chiloé: la visión de tres comunidades en su proceso de reivindicación territorial a través de la Ley Lafkenche (20.249). Memoria (para optar al título profesional de Antropóloga Social) Facultad de Ciencias Sociales, Universidad de Chile, Santiago, 2015.

SIMÕES, Eliane. Territórios em disputa: do impasse ao Jogo Compartilhado entre técnicos e residentes no Parque Estadual da Serra do Mar. São Paulo: Annablume, FAPESP, 2015.

SKEWES, Juan Carlos. (En prensa). El renuevo de la vida, la ética ambiental y la protec- 
Francisco Araos, Emilia Catalán, Ricardo Álvarez, David Núñez, Francisco Brañas, Wladimir Riquelme

ción de los animales: una doble lectura a partir de la celebración del rodeo como parte de la liturgia nacionalista. En: PHILIPPI JR., Arlindo; SAMPAIO, Carlos Alberto Cioce; FLORIT, Luciano (Org.). Ética Socioambiental.

SKEWES, Juan Carlos; ÁLVAREZ, Ricardo; NAVARRO, Magdalena. Usos consuetudinarios, conflictos actuales y conservación en el borde costero de Chiloé insular. Revista Magallania, v. 40, n. 1, p. 109-125, 2012.

SUCKEL, Hanny; RAZETO, Jorge. Plan de manejo comunitario Complejo el Zaino-Laguna Copín. San Felipe: Ediciones Almendral, 2016.

TECKLIN, David. La apropiación de la costa chilena: ecología política de los derechos privados en torno al mayor recurso público del país. En: PRIETO, Manuel; BUSTOS, Beatriz; BARTON, Jonathan (Eds.). Ecología política en Chile: naturaleza, propiedad, conocimiento y poder. Santiago: Editorial Universitaria, 2016. p. 121-142.

THER, Francisco. Diversidad y sentido patrimonial: contribuciones desde la antropología del territorio al estudio de las comunidades tradicionales. Terra Plural, v. 5, n. 2, p. 153-167, 2012.

THER, Francisco. Antropología del territorio. Polis - Revista de la Universidad Bolivariana, V. 2, n. 32, p. 493-510, 2012.

VARGAS, C.; DONOSO, G.; VÉLEZ, D.; DONOSO, D.; CHEUQUEMAN, M.; ÑANCUCHEO, C.; MILLABUR, A.; HIRIART-BERTRAND L. Espacios Costeros Marinos de Pueblos Originarios: historia, desafíos y oportunidades para el desarrollo del borde costero en Chile. En: Libro de Resúmenes XXXVI Congreso de Ciencias del Mar. Sustentabilidad y Multidisciplina en Ciencias del Mar SUMAR, Concepción, Chile. 2016. Disponible en: http://studylib.es/doc/7314016/libro-de-res\%C3\%BAmenes---ciencias-del-mar-2016. Acceso el: 09 abr. 2019.

WEST, Paige; IGOE, James; BROCKINGTON, Dan. Parks and peoples: the social impact of protected areas. Annual Review of Anthropology, Annual Reviews, v. 35, n. 1, p. 251$277,2006$. 